\title{
Pressure-driven flow focusing of two miscible liquids
}

\author{
Ilyesse Bihi, ${ }^{1}$ Doriane Vesperini, ${ }^{1}$ Badr Kaoui, ${ }^{1}$ and Anne Le Goff ${ }^{1, *}$ \\ ${ }^{1}$ Biomechanics and Bioengineering Laboratory (UMR CNRS 7338), \\ Université de Technologie de Compiègne, Alliance Sorbonne Université \\ 60200 Compiègne, France
}

(Dated:)

\begin{abstract}
Flow focusing consists in injecting a core liquid into another surrounding flowing sheath liquid. Here we investigate experimentally the influence of imposing pressure to generate coflow of two miscible liquids. We inject water in the central inlet of a cross-junction microfluidic device and different mixtures of glycerol-water in the two lateral inlets. A pressure generator is used to control the flows and the established flow rates are monitored in both inlets. We draw a state diagram that delimits the regions of the coflow, the inner and outer back flows. We measure the width of the jet as a function of different control parameters: the inlet pressures, the flow rates, the viscosity contrast and the channel aspect ratio. We show that the jet width can be controlled by tuning the internal to external pressure ratio solely, provided that the viscosity contrast is low. We discuss the possibility to use such a system to center particles in a channel.
\end{abstract}

\section{INTRODUCTION}

Flow focusing consists in injecting a central liquid (the core) into another second enveloping liquid (the sheath). If the core is more viscous than the external fluid, it is extruded by the sheath and forms a thread moving in a solid-like manner [1]. Else, when the sheath is more viscous, large gradients are present in the inner fluid, that flows in a jet-like manner. Flow focusing of immiscible fluids is encountered in many situations, to generate foams [2], or, more importantly, emulsions [3]. Microfluidic droplet generators have been extensively studied in the last two decades due to the rapid development of droplet-based microfluidic analysis platforms for chemistry and biology [4]. Much less is known about the coflow of miscible liquids, although it is used in applications such as fiber production [5]. The interface between a viscous thread and the surrounding liquid has been shown to undergo hydrodynamic instabilities, leading to a diversity of flow patterns [6-8]. More recently, a study performed on the jet configuration led to similar observations, with three distinct regimes: diffusive, stable and unstable. The jet width was found to follow a scaling law [9]. These results were obtained in a non-deformable, square cross-section microchannel, with flow-rate driven flow.

Conventionally, liquid flow in microfluidic devices is generated using peristaltic or syringe pumps, while pressure control is preferred for gas, that are compressible [10]. Although pumps allow an accurate control of the flow rate in the channels, they suffer from limitations. For example, they may fail to deliver a continuous flow at low flow rates $[11,12]$. Pressure control solutions were developed more recently to overcome these difficulties. Comparison between the efficiency of pressure and flowrate driven pumping has been mostly investigated within

\footnotetext{
* corresponding author: anne.le-goff@utc.fr
}

the context of droplet generation. In the dripping regime for non-miscible flows, scaling laws are found between droplet size (or droplet production frequency) and the flow rate ratio, while no similar laws are obtained with the pressure ratio [13]. In spite of this, the physical phenomena governing droplet formation are the same in flowrate controlled and pressure controlled experiments [14], and pressure-controlled droplet generation keeps being investigated, since syringe pump fluctuations have been shown to increase polydispersity in flow rate-controlled experiments [15]. However, the above studies have considered immiscible coflowing liquids while many questions remain unanswered for the case of miscible liquids.

Flow focusing of miscible fluids can be used to focus particles in microchannels [16]. In most of these systems, there is a need to control the concentration of the particle suspension infused in the inlet. If the density of particles does not perfectly match that of the solvent, sedimentation occurs in the suspension. It can be slowed down by tuning the density and viscosity of the suspending fluid [17], but it is usually prevented by agitating the reservoir. Combining mechanical agitation and flow rate control implies to either set the whole syringe pump into motion [18], or agitate the suspension and use a peristaltic pump [19]. The former solution is cumbersome and the latter is not appropriate when the suspension flow rate is low, as few pumps are able to deliver pulsefree flows at low flow rates. A pressure controller and a gentle mechanical agitation of the suspension reservoir is a convenient way to achieve a steady particle throughput, even at low flow rate. There is therefore a need to better understand the behavior of pressure-driven miscible coflows.

In this work, we study experimentally the coflow of two miscible liquids in a cross-junction microfluidic device using a pressure-driven flow. The experimental setup is described in section II. In section III, we build a state diagram describing the behavior of the system. The width of the jet is studied in section IV, and microcapsule centering is presented in section $\mathrm{V}$ as an example of application. 
(a)

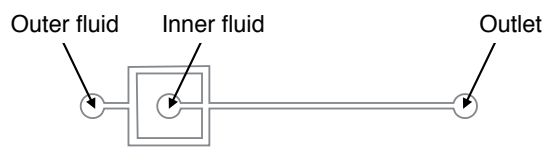

(b)

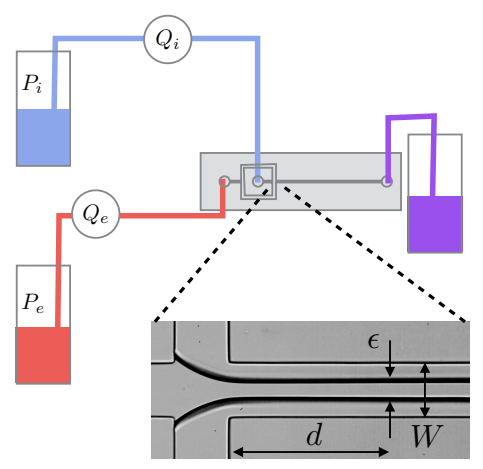

FIG. 1. (a) Sketch of the microfluidic channel. (b) Sketch of the whole experimental setup, where two miscible liquids are injected with a pressure controller into the microfluidic crossjunction. The two liquids have different viscosities: $\mu_{\mathrm{e}}$ and $\mu_{\mathrm{i}}$. The jet width $\epsilon$ is measured at a distance $d$ downstream from the cross-junction. The channel width is $W$.

\section{EXPERIMENTAL SET-UP}

Microfluidic devices are fabricated in polydimethylsiloxane (PDMS) using the standard soft lithography technique and sealed to a glass slide using a surface plasma treatment [20, 21]. Two microchannels with the same width $W$ intersect perpendicularly to create a crossjunction configuration, as illustrated in Figure 1. All devices have a uniform height $H=50 \mu \mathrm{m}$ and different widths $W=50,100$ and $200 \mu \mathrm{m}$. This corresponds to aspect ratios of $a=\frac{W}{H}=1,2$ and 4 , respectively.

We introduce deionized water into the central channel (the core flow) and a glycerol-water mixture in the two side channels (the sheath flow). The dynamic viscosity of the internal liquid is constant $\mu_{\mathrm{i}}=1 \mathrm{cP}$, while of the glycerol-water mixtures spans a range of viscosity $1 \mathrm{cP} \lesssim \mu_{\mathrm{e}} \lesssim 300 \mathrm{cP}$ by tuning the concentration of the added glycerol. These correspond to viscosity contrasts of $\chi^{-1}=\frac{\mu_{\mathrm{e}}}{\mu_{\mathrm{i}}}=1$ to 300 . Viscosity is measured at $20^{\circ}$ for each experiment using a cone-plate viscometer (Haake, Thermofisher Scientific).

In this setup, the control parameters are the pressures in the reservoirs of the core liquid $P_{\mathrm{i}}$ and of the sheath liquid $P_{\mathrm{e}}$, imposed by a microfluidic flow control system (MFCS-EZ, Fluigent) and varied between 0 and 2000 mbar with respect to the atmospheric pressure $p^{*}$. For each imposed pressure, we measure the core and sheath flow rates $Q_{\mathrm{i}}$ and $Q_{\mathrm{e}}$ using a flow sensor (Flowell, Fluigent). We use $250 \mu \mathrm{m}$ i.d. polyethertetherketone (PEEK) tubing to connect the reservoirs to the flow sen- sors, the flow sensors to the chip, and to collect liquid from the outlet. All segments are $30 \mathrm{~cm}$ long.

The whole microfluidic device is placed on the stage of an inverted microscope (Leica DMI8) equipped with a CCD camera (Photron SA3) to visualize the coflow region. We measure the jet width $\epsilon$ at a distance $d=$ $6 \times H$ from the coflow junction.

\section{JET GENERATION}

We initially set equal pressures at the two inlets $\left(P_{\mathrm{i}}=\right.$ $\left.P_{\mathrm{e}}\right)$ and get a stable coflow. We then decrease the external pressure $P_{\mathrm{e}}$, by increments of $50 \mathrm{mbar}$, until the coflow vanishes and the inner fluid starts invading the external fluid inlet. At this moment, $P_{\mathrm{e}}=P_{\mathrm{e}, \min }$. The transition to this regime, called inner backflow, is indicated by filled squares in Figure 2(a). From there, $P_{\mathrm{e}}$ is increased again with the same increments until it reaches the limit of the outer backflow $P_{\mathrm{e}, \max }$, at which the external fluid invades the inner fluid inlet. Figure 2(b) displays the flow regimes, where the arrows indicate the direction of the flow.

In the regime of stable coflow, we observe a jet of the inner fluid in the external fluid. Since the two fluids are miscible, the interface is defined as a region where a gradient in glycerol concentration is present. This gradient is steep when the two liquids meet at the cross-junction and it smears out as fluids flow in the main channel due to the diffusion between glycerol and water. The Péclet number Pe, comparing the importance of advection and diffusion, is here defined as:

$$
\mathrm{Pe}=\frac{Q_{\mathrm{i}}+Q_{\mathrm{e}}}{H \mathrm{D}} .
$$

with $\mathrm{D}=10^{-5} \mathrm{~cm}^{2} / \mathrm{s}$ the diffusion coefficient of glycerol in water [22]. The distance beyond which the two fluids are perfectly mixed increases with Pe. At low Péclet numbers $(\mathrm{Pe}<860$ for square cross-section channels), the flow is dominated by diffusion [9]. Here, the Schmidt number $\mathrm{Sc}=\mu_{e} /\left(\rho_{e} D\right)$ is always larger than 5000, indicating that diffusive mass transfer is negligible with respect to diffusive momentum transfer. This is in agreement with the fact that we do not observe diffusive instabilities [23]. In our experimental conditions, the Péclet number Pe remains within the range of $2-30 \times 10^{3}$; therefore, the system operates in the convective regime, as seen in Figure 2(c). Although our field of view is located close to the cross-junction, as sketched in Figure 1, at these values of Pe, the interface is maintained thanks to convection to a certain distance.

It is found that the two limits of the coflow regime correspond to straight lines. These limits can pe predicted using the electrical analogy represented in Figure 3 [24].

The Poiseuille law that relates the flow rate $Q$ directly to the pressure drop $\Delta P$,

$$
\Delta P=R Q,
$$




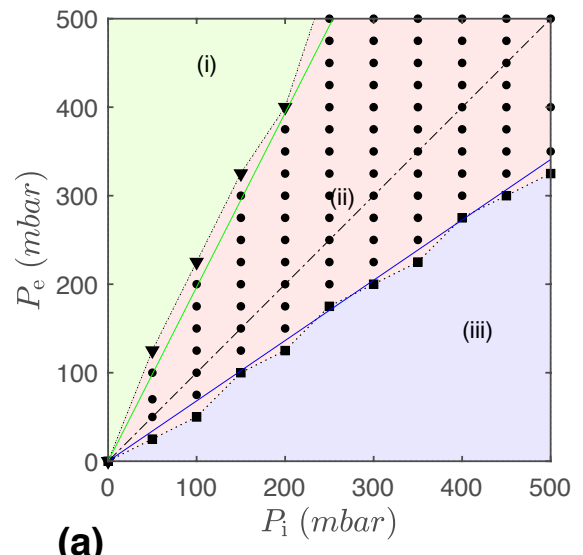

(a)
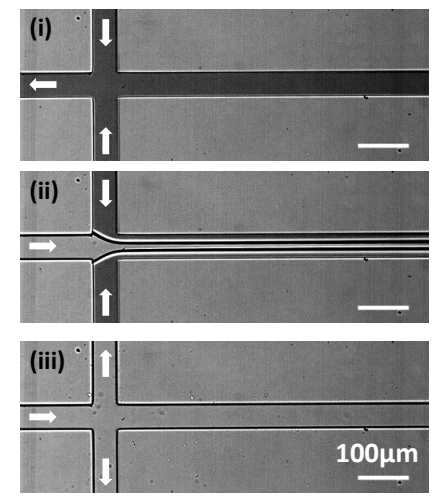

(b)

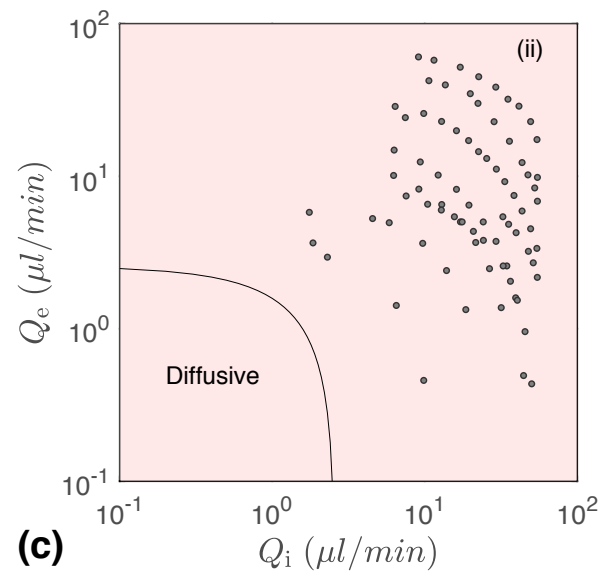

(c)

$Q_{\mathrm{i}}(\mu l / \min )$

FIG. 2. (a) The region of existence and stability of the coflow of water (inner liquid) into glycerol-water mixture (outer liquid). Filled circles correspond to stable coflow, while filled triangles and filled squares respectively represent the onset of backflows in the inner and outer inlets. The solid lines are borders computed theoretically by equations (6) and (7). (b) Micrographs showing the three obtained flow regimes: (i) backflow in the inner inlet, (ii) stable coflow and (iii) backflow in the outer inlet. The aspect ratio is $a=1$. The viscosity ratio is $\chi^{-1}=\frac{\mu_{\mathrm{e}}}{\mu_{\mathrm{i}}}=8$. Scale bar $50 \mu \mathrm{m}$. (c) The measured flow rates of the data shown in (a).

where $R$ is the hydraulic resistance, yields the following relationships between $P_{\mathrm{e}}, P_{\mathrm{i}}, Q_{\mathrm{e}}$ and $Q_{\mathrm{i}}$ :

$$
\begin{gathered}
P_{\mathrm{i}}=R_{i} Q_{\mathrm{i}}+R_{s}\left(Q_{\mathrm{i}}+Q_{\mathrm{e}}\right), \\
P_{\mathrm{e}}=R_{e} Q_{\mathrm{e}}+R_{s}\left(Q_{\mathrm{i}}+Q_{\mathrm{e}}\right),
\end{gathered}
$$

where $R_{i}, R_{e}$ and $R_{s}$ are the hydraulic resistances of the core liquid inlet, the sheath liquid inlet and the outlet, respectively. In writing equations (3) and (4), we assume that $Q_{s}=Q_{i}+Q_{e}$, we neglect the contraction of the water-glycerol mixture. By doing so, we introduce an error on the specific gravity of the mixture, and thus on $Q_{s}$, of order 1\% [25]. For a more accurate estimate of $Q_{s}$, we could implement a model linking glycerol fraction

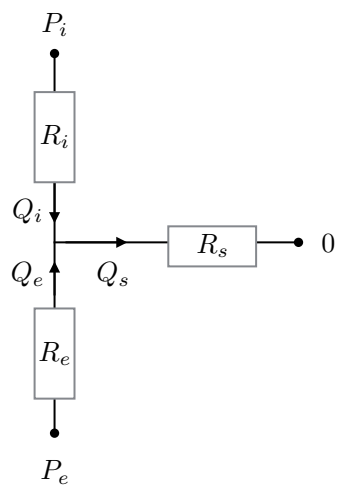

FIG. 3. The electric circuit analogy to our the fluidic experimental setup network. The indexes (i, e and s) refer to the core, the sheath and the outflow liquids. and density, such as the one presented in [26]. Here, we neglect the gycerol contraction and this uncertainty is taken into account in the calculations as an additional source of error on the inlet pressures $P_{i}$ and $P_{e}$.

As both the flow through the PDMS chip and through the capillary pipes contribute to the total pressure drop, each of the hydraulic resistances is expressed as the sum of two terms. The first is the resistance of the rectangular channels in the microfluidic device. The second term represents the resistance in the capillary tubes transporting the liquid into and out of the microfluidic chip. Thus, following Ref. [27] the resistance can be expressed as:

$$
\begin{aligned}
R_{\alpha}= & \underbrace{\frac{12 \mu_{\alpha} L_{\alpha}}{H^{3} W}\left[1-\sum_{n, o d d}^{\infty} \frac{192}{n^{5} \pi^{5}} \frac{H}{W} \tanh \left(\frac{n \pi W}{2 H}\right)\right]^{-1}}_{\text {channels }} \\
& +\underbrace{\frac{8 \mu_{\alpha} l}{\pi r^{4}}}_{\text {tubes }}
\end{aligned}
$$

where the index ( $\alpha=\mathrm{i}$, e and $\mathrm{s}$ ) refer to the core, the sheath and the outflow liquids, see Figure 3. $l$ and $r$ are the length and the radius of the tubes upstream the microfluidic chip, which are the same in the three branches. $L_{\alpha}, H$ and $W$ are the length, the height and the width of the channels in the microfluidic device. The channel depth $H$ is uniform in the whole device, and all the channels have a width $W=a H$. The length of the internal fluid inlet and the outlet are $L_{i}=1.2 \mathrm{~mm}$ and $L_{s}=4.7 \mathrm{~mm}$ respectively. For the entrance of the external fluid, as the initial channel of length $L_{1 e}=320 \mu \mathrm{m}$ divides into two branches of length $L_{2 e}=6.9 \mathrm{~mm}$ that meet again at the cross-junction, the hydraulic resistance 


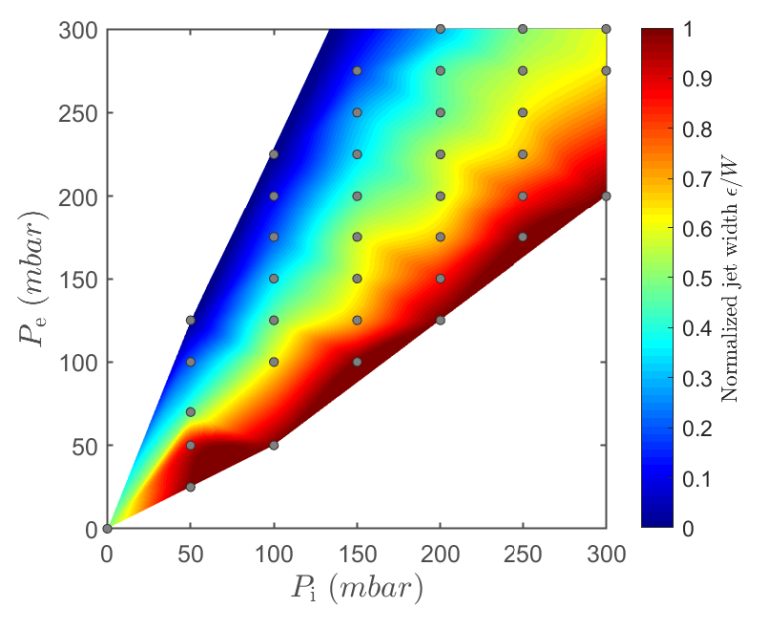

FIG. 4. Scaled jet width $\epsilon / W$ in the coflow regime of the flow diagram $\left(P_{\mathrm{i}}, P_{\mathrm{e}}\right)$. The aspect ratio is $a=1$ and the viscosity contrast is $\chi^{-1}=8$.

of this branch is equal to that of a single channel of length $L_{e}=L_{1 e}+L_{2 e} / 2$. This quantity is used in equation (5) to compute $R_{e}$. For an aspect ratio $a=1$ and a viscosity contrast $\chi=1 / 8$, the inlet hydraulic resistances are approximately $R_{i} \approx 1.2 \times 10^{13} \mathrm{~kg} \mathrm{~m}^{-4} \mathrm{~s}^{-1}$ and $R_{e} \approx 1.9 \times 10^{14} \mathrm{~kg} \mathrm{~m}^{-4} \mathrm{~s}^{-1}$. For the least resistive of the channels $(a=4)$, the capillary PEEK tubes account for about $80 \%$ of $R_{i}$ and $55 \%$ of $R_{e}$. Because the outflow is a mixture of the inner and the outer liquids, its viscosity is expected to vary between $\mu_{\mathrm{i}}$ and $\mu_{\mathrm{e}}$. Thus, $R_{s}$ can not be determined exactly. However, in the limits of the coflow regime, the outflow consists of either pure inner or pure outer liquid. The computed value is $R_{s} \approx 2.0 \times 10^{14} \mathrm{~kg} \mathrm{~m}^{-4} \mathrm{~s}^{-1}$ in the former case, and $R_{s} \approx 1.6 \times 10^{15} \mathrm{~kg} \mathrm{~m}^{-4} \mathrm{~s}^{-1}$ in the latter.

By substituting either $Q_{\mathrm{i}}$ or $Q_{\mathrm{e}}$ by zero in Eqs. (3) and (4), we get expressions for the upper limit $P_{\mathrm{e}, \max }$ and for the lower limit $P_{\mathrm{e} \text {,min }}$ between which the coflow is possible for a given pressure $P_{i}$ in the water inlet:

$$
\begin{aligned}
& P_{\mathrm{e}, \max }=P_{\mathrm{i}} \frac{R_{e}+R_{s}}{R_{s}}, \\
& P_{\mathrm{e}, \min }=P_{\mathrm{i}} \frac{R_{s}}{R_{i}+R_{s}} .
\end{aligned}
$$

The solid lines in Figure 2(a) depict the boundaries of the coflow regime plotted using these equations. A good agreement is observed between the model and the experimental data. In the following section, we study the width of the jet obtained in stable coflow conditions. This means that $P_{\mathrm{e}, \min }<P_{\mathrm{e}}<P_{\mathrm{e}, \max }$, but also that the fluid velocity is both large enough so that the miscible interface between the two fluids remains sharp enough to be observed by the microscope, but small enough to avoid the instabilities that appear at high flow rates [9].

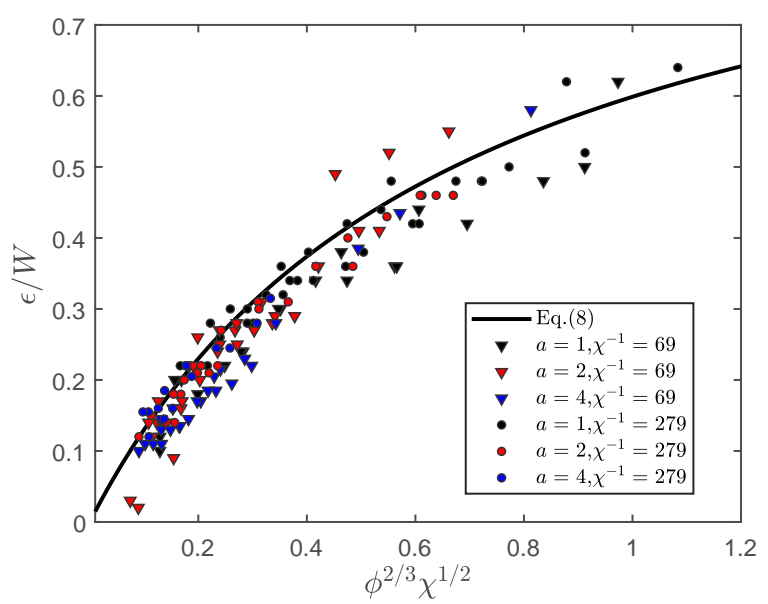

FIG. 5. The scaled jet width $\epsilon / W$ as a function of $\phi^{2 / 3} \chi^{1 / 2}$. Three microfluidic cross-junctions with different aspect ratios have been studied $a=1,2,4$. Two fluid pairs are depicted with viscosity contrasts $\chi^{-1}=69$ and 279 . The solid line represents Eq. (8).

\section{JET WIDTH}

We characterize the jet width $\epsilon$ as a function of the viscosity contrast $\chi=\mu_{\mathrm{i}} / \mu_{\mathrm{e}}$, the aspect ratio $a=W / H$ and the inlet flow pressures $\left(P_{\mathrm{i}}, P_{\mathrm{e}}\right)$. We show in Figure 4 that the scaled jet width $\epsilon / W$, here plotted for $\chi^{-1}=8$ and $a=1$, is highly variable in the region of stable coflow. For a fixed pressure $P_{\mathrm{i}}$ in the inner inlet, increasing the external pressure $P_{\mathrm{e}}$ results in a decrease of the jet width.

Using a Hele-Shaw approximation, the jet width can be written as $\epsilon / W=\left[1+(\phi \chi)^{-1}\right]^{-1}$, where $\phi=Q_{\mathrm{i}} / Q_{\mathrm{e}}$ is the flow rate ratio [7]. When the cross-section of the channel is square, the effect of the side walls are significant and lead to the empirical formula [9]

$$
\epsilon / W \approx \frac{1}{1+0.67 \phi^{-2 / 3} \chi^{-1 / 2}}
$$

Equation (8) is compared with experimental data in Figure 5, where we plot $\epsilon / W$ versus $\phi^{-2 / 3} \chi^{-1 / 2}$. Three aspect ratios $(a=1,2,4)$ and two viscosity contrasts, $\chi^{-1}=69$ and 279 , have been used. In every set of experiments, we observe a monotonic increase of the jet width when the flow rate ratio increases and the experimental data show a good agreement with equation (8). Here, scaling by $W$ allows the collapse of all the experimental data into one single curve. We find that the scaled jet width $\epsilon / W$ does not vary despite the aspect ratio have been changed from 1 to 4 .

In order to relate Eq. (8) to our control parameters, we express the flow rate ratio as a function of inlet pressures and hydraulic resistances using Eqs. (3) and (4): 


$$
\phi=\frac{Q_{\mathrm{i}}}{Q_{\mathrm{e}}}=\frac{R_{s}-\left[R_{e}+R_{s}\right]\left(\frac{P_{\mathrm{i}}}{P_{\mathrm{e}}}\right)}{-R_{i}-R_{s}+R_{s}\left(\frac{P_{\mathrm{i}}}{P_{\mathrm{e}}}\right)}
$$

The fluid flowing out of the cross junction is a mixture of two liquids in variable proportions. The hydraulic resistance $R_{s}$ is therefore likely to vary with the flow rate ratio and the pressure ratio.

In Figure 6(a), we plot the flow rate ratio $\phi$ as a function of the pressure ratio $P_{i} / P_{e}$, for $a=2$ and $\chi^{-1}=279$. Raw experimental data (filled circles) are superimposed with Eq. (9), where $R_{s}$ has been estimated using Eq. (5) for the two limiting cases $\mu_{\mathrm{s}}=\mu_{\mathrm{i}}$ (solid line) and $\mu_{\mathrm{s}}=\mu_{\mathrm{e}}$ (dashed line). The graph is on a log-linear scale, as $\phi$ spans two orders of magnitude whereas $P_{i} / P_{e}$ only increases by a factor 2. Experimental values are located between the two limiting curves, because each data point corresponds to a different value of $R_{s}$. Also, the curves obtained for different values of $P_{i}$ do not collapse on a single master curve: there is no bijection between $P_{i} / P_{e}$ and $\phi$. This explains why the jet width $\epsilon$, that is controlled by the flow rate ratio, not only depends on the pressure ratio but also on the absolute value of the pressure, as illustrated in Figure 6(b) and quantified in Figure 6(c).

At low viscosity contrast $\left(\chi^{-1}=8\right)$, when we plot again $\phi$ versus $P_{i} / P_{e}$ in Figure $7($ a), the gap between the two limiting curves gets narrower and the data points are less dispersed. This implies that the flow rate ratio $\phi$ varies on a much smaller range than in Figure 6(a) for a given value of the pressure ratio $P_{i} / P_{e}$. As a consequence, the jet width $\epsilon$ is less sensitive to variations of the absolute value of $P_{i}$, as can be observed in Figure 7(b). This is reported more quantitatively in Figure 7(c). For two values of the pressure ratio $P_{i} / P_{e}$, we show that the jet width $\epsilon$ does not depend on $P_{i}$. At low viscosity contrast, the jet width can be controlled by tuning the pressure ratio, independently of the absolute value of the pressures.

In some of the conditions explored in this article, relatively small pressure variations could lead to significant changes in the flow rate ratio, which was found to govern the jet width. This observation is consistent with observations made with non-miscible fluids in the context of producing emulsions. The domain of steady droplet generation appears wide in a $\left(Q_{i}, Q_{e}\right)$ diagram and much narrower in a $\left(P_{i}, P_{e}\right)$ diagram [14]. In order to achieve a good flow control using a pressure controller, it is necessary to take into account the pressure drop in the capillary tubing, that may exceed the pressure drop in the microfluidic channel, and to compute the flow rate ratio as a function of the control parameters. The pressure drop in the capillaries may be reduced by increasing their lumen, if the dead volume is not a limiting factor, and keeping the connecting tubes as short as possible. But the pressure drop in the capillaries can also be used as an advantage. If the hydraulic resistances are large enough in the inlets, then $P_{\mathrm{i}} \simeq R_{i} Q_{\mathrm{i}}$ and $P_{\mathrm{e}} \simeq R_{e} Q_{\mathrm{e}}$, which means that the flow rate ratio becomes

$$
\phi \simeq \frac{R_{e}}{R_{i}} \frac{P_{\mathrm{i}}}{P_{\mathrm{e}}}
$$

In this limit, just like in the limit of low viscosity contrast, the pressure ratio can be used to control the jet shape. The pressure drop in the connecting tubes is often overlooked by users of pressure controllers, who sometimes omit to mention their dimensions. Our results show that this pressure drop is significant and can be exploited to achieve a good control of the experiment.

\section{APPLICATION: CENTERING OF MICROCAPSULES}

In earlier work, we used a flow focusing module to center microcapsules at the entrance of a microfluidic sorter [28]. We show in Figure 8 measurements of the offcentering $\delta$, defined as the distance between the capsule center and the channel axis, about 10 capsule diameters downstream of the flow focusing. Capsules are infused at variable pressure $P_{i}$ and the surrounding glycerol at $P_{e}=5 P_{i}$. We see in Figure 8(a) that $\delta$ decreases with the inlet pressure. The scattering of centering data also decreases with $P_{i}$, as seen in Figure 8(b). This improved centering at high pressures is due to a thinner jet, as discussed in previous paragraph. This effect is likely to be amplified by fluid-structure interaction [29]. In all the experimental conditions explored here, the off-centering $\delta$ remains inferior to $5 \mu \mathrm{m}$, which is well below the channel width $w=200 \mu \mathrm{m}$. This indicates that pressurecontrolled flow focusing is an efficient centering method. Depending on the application, the centering may require a constant value of $\epsilon$. In this case, even when a pressure controller is used, it would be advisable to define target values of the inner and outer flow rates and to use a feedback loop to adjust the pressures, a possibility that is now offered by some pressure controllers, or to choose long, resistive capillary tubes to connect the reservoirs to the chip, and reach the conditions of validity for equation (10).

\section{CONCLUSIONS}

We have reported a systematic study about controlling the flow of two miscible liquids using a pressure controller. We have used cross-junction microfluidic devices with different aspect ratios and we have carried out experiments using liquids with different viscosity contrasts. We have identified and characterized the challenges associated with the pressure control of miscible coflows, in contrast to the flow rate controller. The main encountered drawbacks are the risk of backflow in the inlets, and the lack of simple scaling laws to predict the jet width as a function of the inlet pressures. A simple analysis of 


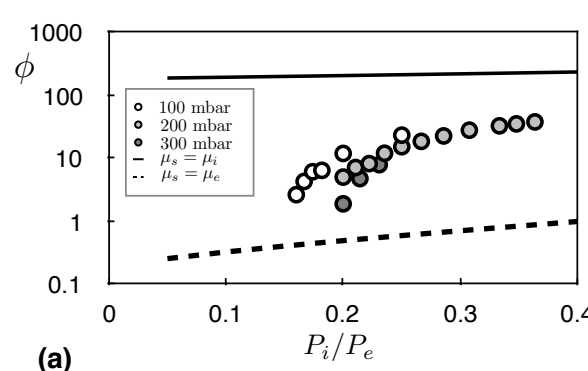

(a)

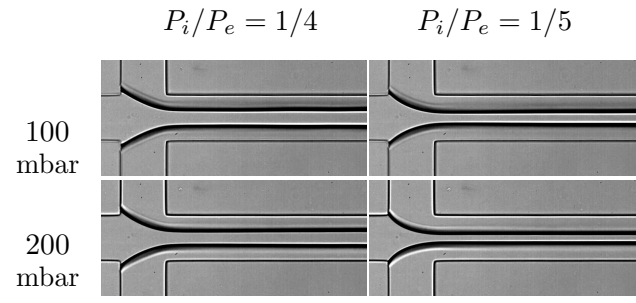

(b)

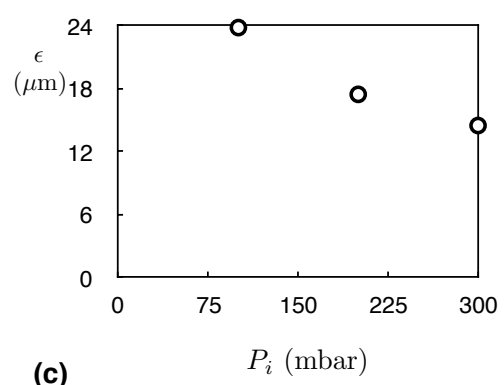

(c)

FIG. 6. (a) Flow rate ratio $Q_{\mathrm{i}} / Q_{\mathrm{e}}$ as a function of the pressure ratio $P_{\mathrm{i}} / P_{\mathrm{e}}$ between the inner and the outer fluids, for a viscosity constrast $\chi^{-1}=279$ and an aspect ratio $a=2$. The solid and the dashed line depict Eq.( 9$)$ using respectively the internal viscosity $\mu_{\mathrm{i}}$ and the external viscosity $\mu_{\mathrm{e}}$ to estimate $R_{s}$. (b) Micrograph showing the shape of the jet for an inner pressure $P_{i}$ of 100 and 200 mbar and two values of the pressure ratio. (c) Jet width as a function of the water inlet pressure $P_{i}$, for a constant pressure ratio $P_{i} / P_{e}=1 / 4$.

(a)

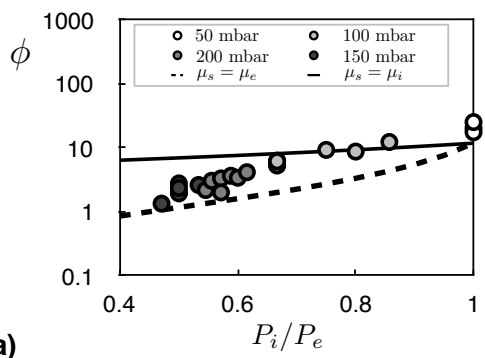

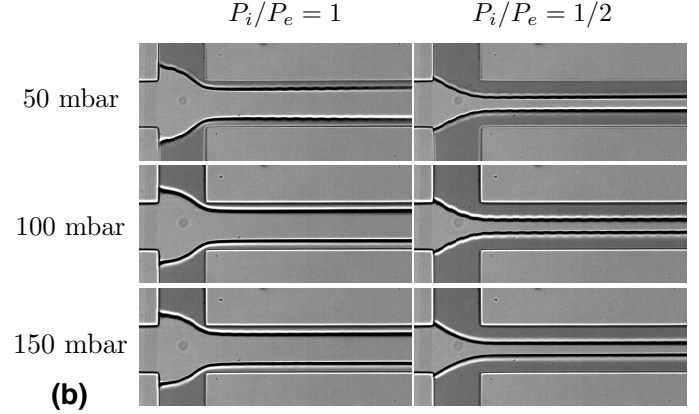

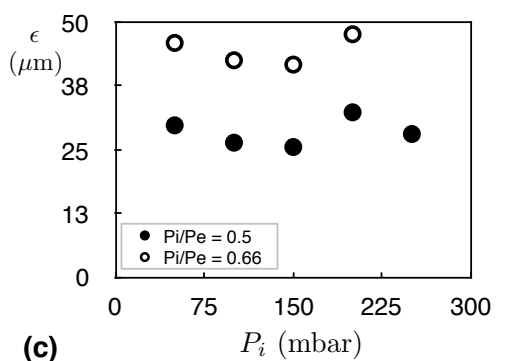

FIG. 7. (a) Flow rate ratio $Q_{\mathrm{i}} / Q_{\mathrm{e}}$ as a function of the pressure ratio $P_{\mathrm{i}} / P_{\mathrm{e}}$ between the inner and the outer fluids, for a viscosity constrast $\chi^{-1}=8$ and an aspect ratio $a=2$. The solid and the dashed line depict Eq.( 9) using respectively the internal viscosity $\mu_{\mathrm{i}}$ and the external viscosity $\mu_{\mathrm{e}}$ to estimate $R_{s}$. (b) Micrograph showing the shape of the jet for an inner pressure $P_{i}$ between 50 and 150 mbar and two values of the pressure ratio. (c) Jet width as a function of the water inlet pressure $P_{i}$.

the channel geometry, together with the knowledge of the fluid viscosities, allows to predict the shape of the stability domain of the jet and to avoid backflows. In particular, the resistivity of the capillaries connecting the fluid reservoirs to the chip must be taken into account early in the conception of the microfluidic circuit, and can be adjusted to make the system less sensitive to pressure fluctuations.

\section{ACKNOWLEDGEMENTS}

We gratefully acknowledge the financial support from the Hauts-de-France region and the European Regional Development fund (FEDER) entitled "FORPLAQ". DV acknowledges funding from the French Ministry of Research and Higher Education.
[1] T. Cubaud and T. G. Mason, New Journal of Physics 11, 075029 (2009).

[2] P. Garstecki, I. Gitlin, W. DiLuzio, G. M. Whitesides, E. Kumacheva, and H. A. Stone, Applied Physics Letters 85, 2649 (2004).

[3] S. L. Anna, N. Bontoux, and H. A. Stone, Applied physics letters 82, 364 (2003).

[4] O. J. Dressler, X. Casadevall i Solvas, and A. J. deMello, Annual Review of Analytical Chemistry 10, 1 (2017), pMID: 28375703.

[5] C. M. Hwang, A. Khademhosseini, Y. Park, K. Sun, and S.-H. Lee, Langmuir 24, 6845 (2008).
[6] T. Cubaud and T. G. Mason, Physics of Fluids 20, 053302 (2008).

[7] T. Cubaud and T. G. Mason, Physical Review E 78, 056308 (2008).

[8] O. Bonhomme, J. Leng, and A. Colin, Soft Matter 8, 10641 (2012).

[9] X. Hu and T. Cubaud, Phys. Rev. Fluids 1, 044101 (2016).

[10] B. Laborie, F. Rouyer, D. E. Angelescu, and E. Lorenceau, Physics of Fluids 28, 063103 (2016).

[11] Z. Li, S. Y. Mak, A. Sauret, and H. C. Shum, Lab on a Chip 14, 744 (2014). 
[12] W. Zeng, I. Jacobi, D. J. Beck, S. Li, and H. A. Stone, Lab on a Chip 15, 1110 (2015).

[13] T. Ward, M. Faivre, M. Abkarian, and H. A. Stone, Electrophoresis 26, 3716 (2005).

[14] S. Lignel, A.-V. Salsac, A. Drelich, E. Leclerc, and I. Pezron, Colloids and Surfaces A: Physicochemical and Engineering Aspects 531, 164 (2017).

[15] W. Zeng, I. Jacobi, S. Li, and H. A. Stone, Journal of Micromechanics and Microengineering 25, 115015 (2015).

[16] X. Xuan, J. Zhu, and C. Church, Microfluidics and Nanofluidics 9, 1 (2010).

[17] M. Faivre, M. Abkarian, K. Bickraj, and H. A. Stone, Biorheology 43, 147 (2006).

[18] A. G. Håti, D. C. Bassett, J. M. Ribe, P. Sikorski, D. A. Weitz, and B. T. Stokke, Lab on a Chip 16, 3718 (2016).

[19] A. Blin, A. Le Goff, A. Magniez, S. Poirault-Chassac, B. Teste, G. Sicot, K. A. Nguyen, F. S. Hamdi, M. Reyssat, and D. Baruch, Scientific Reports 6, 21700 (2016).

[20] Y. Xia and G. M. Whitesides, Annual review of materials science 28, 153 (1998).

[21] G. M. Whitesides, E. Ostuni, S. Takayama, X. Jiang, and D. E. Ingber, Annual review of biomedical engineering 3, 335 (2001).

[22] W. M. Haynes, CRC handbook of chemistry and physics (CRC press, 2014).

[23] T. Cubaud and S. Notaro, Physics of Fluids 26, 122005 (2014).

[24] K. W. Oh, K. Lee, B. Ahna, and E. P. Furlani, Lab on a Chip 12, 515 (2012).

[25] L. W. Bosart and A. O. Snoddy, Industrial \& Engineering Chemistry, Industrial \&

(a)
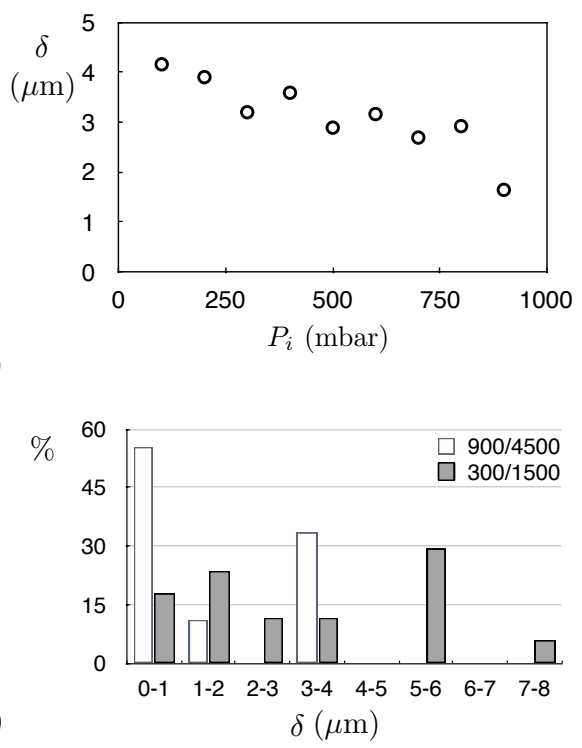

FIG. 8. (a) Microcapsule centering using miscible flow focusing. A microcapsule suspension is injected in the central inlet of a flow focusing channel. Glycerol is used as the sheath fluid. The pressure ratio $P_{\mathrm{i}} / P_{\mathrm{e}}=1 / 5$ is constant and $P_{\mathrm{i}}$ varies between 100 and 900 mbar. Downstream of the flow focusing, the distance $\delta$ between the capsule center and the channel axis is measured. (b) Distribution of off-centering $\delta$ for two values of $P_{\mathrm{i}}$.
Engineering Chemistry 20, 1377 (1928).

[26] A. Volk and C. J. Kähler, Experiments in Fluids 59 (2018), 10.1007/s00348-018-2527-y.

[27] H. Bruus, Theoretical microfluidics, Vol. 18 (Oxford university press Oxford, 2008).

[28] D. Vesperini, O. Chaput, N. Munier, P. Maire, F. Edwards-Lévy, A.-V. Salsac, and A. Le Goff, Medical Engineering and Physics 48, 68 (2017).

[29] C. Rorai, F. Nason, L. Zhu, G. Casagrande, G. Dubini, and L. Brandt, Procedia IUTAM 16, 41 (2015), iUTAM Symposium on Dynamics of Capsules, Vesicles and Cells in Flow (15-19 July 2014; Compiègne, France). 